\title{
Induction of a transient dysexecutive syndrome in Parkinson's disease using a subclinical dose of scopolamine
}

\author{
M.A. Bédard ${ }^{\mathrm{a}, *}$, S. Lemay ${ }^{\mathrm{a}}$, J.F. Gagnon ${ }^{\mathrm{a}}$, \\ H. Masson ${ }^{\mathrm{b}}$ and F. Paquet ${ }^{\mathrm{a}}$ \\ ${ }^{\mathrm{a}} U Q A M$ and Laboratoire de Neuropharmacologie \\ Cognitive, CHUM, Notre-Dame, Montréal, Canada \\ ${ }^{\mathrm{b}}$ Université de Montréal and Service de neurologie, \\ CHUM, Notre-Dame, Montréal, Canada
}

\begin{abstract}
Parkinson's Disease (PD) is often associated with a subcorticofrontal syndrome (SCFS) that is mainly characterized by executive dysfunctions. The complete biochemistry of these dysfunctions remain misunderstood although many studies have suggested a role of the dopaminergic lesions. However, cholinergic lesions in this disease may also account for the SCFS occurrence. The present study has assessed the effects of an acute subclinical dose of scopolamine in normal controls and in PD patients who were devoid of cognitive deficit. Results indicates that PD patients but not normal controls developed a transient SCFS for the duration of the drug action. In contrast to other populations with cholinergic depletions - such as Alzheimer's disease - cholinergic blockage in PD exacerbates specifically the dysexecutive syndrome without inducing amnesia or sedation. Such a discrepancy between these two neuropsychological profiles are discussed in terms of the specificity of the underlying cholinergic lesions.
\end{abstract}

Keywords: Acetylcholine, frontal syndrome, executive functions, Parkinson's disease, scopolamine, attention, memory, cognition

\section{Introduction}

Parkinson's Disease (PD) is often associated with specific cognitive deficits that include executive dysfunctions such as inattention and a mental inertia, associated with shifting and sequencing dysfunctions and memory impairment in free-recall tasks [51, 53]. Such a constellation of symptoms is frequently referred to as subcortico-frontal syndrome (SCFS), in reference to the locus of lesions in this disease which are though to be principally subcortical, although lesions directly

${ }^{*}$ Corresponding author: Marc-André Bédard, Laboratoire de neuropharmacologie cognitive, CHUM, Pavillon Notre-Dame, 1560, rue Sherbrooke Est, Montréal, Québec, Canada H2L 4M1. E-mail: bedard.marc-andre@uqam.ca. located in the cortex may also be contributive to the syndrome $[4,11,17]$. The expression SCFS remind also the nature of the cognitive deficits which are similar, but less severe, to those observed in patients with frontal lobe lesions. The SCFS can be severe and may reach the DSM-IV criteria for the diagnosis of dementia. It is then recognised as a subcortical dementia [11]. The SCFS must be distinguished from other associated conditions of PD that may affect cognitive functions such as dementia of the Alzheimer type, diffused Lewy Bodies disease or depression, that all shows a high prevalence in PD.

Current knowledge on the physiological basis of SCFS in PD remains unclear. The possibility that the characteristic loss in dopamine (DA) containing neurons of the substantia nigra plays a role has been extensively investigated [15]. These studies were mainly based on the assumption that PD-associated SCFS results from a reduced dopaminergic modulation of the striatum, that disrupts the flow of information within the circuits that link the striatum and the frontal cortex $[3,51]$. The mesocortical dopaminergic degeneration observed in PD has also been suggested as a possible pathogenic factor of SCFS [29]. However, dopaminergic hypotheses cannot explain why treatment of PD with dopaminergic substances (levodopa or dopamine agonists) does not reverse SCFS in these patients [21, 40, 41, 52]. Actually, the more severe are deficits of parkinsonian patients in executive tasks, the lower is their response to dopaminergic treatments [52]. The slight improvement in performance reported with levodopa in some initial studies $[5,33]$ was observed on general intellectual scales and has been mostly attributed to a DA awakening effect (increased vigilance) or to a change in affective state [19, 26, 49]. Comparisons of neuropsychological performance between 'ON' and 'OFF' levodopa conditions were also generally not conclusive. Indeed, the very few changes observed by some authors were generally task-specific in that during 'ON' phases, improvement was observed in some tasks, concurrent with a worsening or lack 
of change in all others [21, 28, 37]. In the study of Lange et al. [31] a withdrawal of levodopa induced a reduction of performances in many tasks sensitive to the SCFS. However, in the latter study, tasks were highly specific and although the performances were improved to a statistical level of significance, they may not be noticeable clinically. Such a phenomenon has been observed in many studies showing that even though the overall SCFS does not respond well to DA treatment, some specific component of the syndrome may improve. For instance, DA treatment of PD were found associated to the improved performances in dual tasks such as working memory tasks $[10,12]$ or visuoauditory concurrent perceptual tasks [34]. It has been suggested that DA depletion in PD may induce a difficulty in sharing attention between two or more concurrent tasks $[34,35]$. Such a hypothesis is in agreement with results obtained from animals with frontal lobe lesions [20] or selective DA depletion [47]. It is not clear however which of the mesocortical or nigrostriatal systems is implicated, although some evidences suggest a critical implication of the latter [16]. In spite of such interesting findings on the relationship between DA and some specific cognitive functioning of the SCFS, it is clear that dopamine dysfunction alone may not account for the overall SCFS of PD.

Among the non-dopaminergic lesions in PD, noradrenergic and serotonergic pathways to the prefrontal cortex have been suggested as possible contributing factors to SCFS, but little evidence supports such a view [7, 15]. Cholinergic systems have also been studied in PD. As in Alzheimer's disease, inominatocortical and septo-hippocampal cholinergic fibres were found to be reduced by 60 to $80 \%$ in parkinsonians with dementia or even in those without dementia $[17,46]$. Other cholinergic systems arising from the tegmental pedunculopontine and laterodorsal nuclei (PPN \& LDN) have also been found to be lesioned in PD [25, 30, 58] but not in Alzheimer's disease. These nuclei are known to have projections namely to the mediodorsal thalamus and to the prefrontal cortex [55] and could therefore play a significant role in the SCFS of PD. The latter hypothesis is supported by the observation that PPN \& LDN cholinergic systems were also found to be lesioned in progressive supranuclear palsy, another neurodegenerative disorder characterized by a severe SCFS [25, 30].

In parkinsonian patients without dementia, the consequences of cholinergic lesions are currently unknown. However, evidences suggest that these lesions could be determinant in the occurrence of SCFS. In opposition to Alzheimer type dementia - in which anticholinergics induce a general worsening of cognitive function with severe memory deficits $[45,50]$ - in PD, these substances specifically affect executive functions associated with SCFS $[6,13,54]$. This suggests that there may exist two different cholinergic mechanisms underlying these two distinct neuropsychological profiles (Alzheimer type and SCFS).

The evolution of the cholinergic lesions in PD may follow those of the nigrostriatal dopaminergic system. Actually, motor symptoms in PD may be present only when dopamine cell death in the substantia nigra reaches a level of 70 to $80 \%$ [27]. This may imply that a long preclinical stage of 7 to 20 years may be present [2]. During these years, some mechanisms of compensation seem to take place in order to withstand the dopamine depletion. Such compensatory mechanisms are not exclusive to the nigro-striatal system and may also occur in cholinergic systems $[1,4]$. A temporary suppression of these cholinergic compensatory mechanisms could be an explanation of the results obtained by Bédard et al. [6] and by Dubois et al. [13], showing that anticholinergic substances given at a subclinical dose - defined as a small dose which remains without detectable cognitive effect in normal subjects - could induce in PD some evidences of a transient SCFS. However, until now, evidence of SCFS was solely based on explicit memory tests. The present study was designed to more accurately describe the phenomenon of cholinergic decompensation that may underly SCFS in PD, by looking at the effect of a subclinical dose of scopolamine on different tasks of executive functions.

\section{Methods}

\subsection{Subjects}

Ten normal controls and 10 patients with a diagnosis of PD were include in the study. Groups were paired for age, sex and education. Control subjects were enrolled from the general population by announcement. Patients were enrolled at the Movement Disorders Clinic of Montreal. Patients were selected on the basis of a screening examination that included neurological, neuropsychological and psychiatric aspects. Criteria for the diagnosis of PD included namely the presence of an akinetic-rigid syndrome that respond well to levodopa. All subjects were required to be free of cognitive deficits on the Mini Mental States (MMS) 
Table 1

Descriptive features of the sample

\begin{tabular}{|c|c|c|c|c|}
\hline & $\begin{array}{c}\text { Parkinsonian } \\
\text { patients } \\
(N=10)\end{array}$ & $\begin{array}{l}\text { Normal } \\
\text { controls } \\
(N=10)\end{array}$ & $t$ & $p$ \\
\hline Age (years) & $69.2(4.8)$ & $68.1(6.6)$ & 0.18 & NS \\
\hline Education (years) & $10.1(3.4)$ & $9.7(2.9)$ & 0.11 & NS \\
\hline Disease duration (years) & $7.1(4.3)$ & - & - & - \\
\hline Disease severity (Hoehn \& Yahr stages) & $2.8(1.7)$ & - & - & - \\
\hline $\operatorname{MMS}^{a}$ (Total score) & $28.7(1.2)$ & $28.3(1.0)$ & 0.00 & NS \\
\hline $\mathrm{DRS}^{b}$ (Mattis) & $138.1(5.6)$ & $140.0(7.2)$ & 0.24 & NS \\
\hline Depression $^{c}$ (GDRS) & $9.5(7.1)$ & $7.9(5.5)$ & 1.07 & NS \\
\hline
\end{tabular}

${ }^{a}$ Mini Mental State examination.

${ }^{b}$ Dementia Rating Scale.

${ }^{c}$ Geriatric Depression Scale.

examination [18] and the Dementia Rating Scale [36]. Performances were also required to be normal on the Boston Naming Test, the Benton Visual Matching Test, the Rey-Osterreith Complex Figure Copy, the Wisconsin Card Sorting Test, the Graphic Sequences of Luria and the Categories and Letters Verbal Fluencies (See [32] for a description of these tests). Subjects with a history of stroke, head injury or neurosurgical operation (including thalamotomy and pallidotomy) were excluded, as were patients with clinical evidence of any psychiatric condition. Depression was ruled out by the administration of the Geriatric Depression Rating Scale. Socio-demographic and clinical data were recorded for each patient and are presented in Table 1. All selected patients were on stable dopaminergic medication ( $l$-dopa: $n=10$; Bromocriptine: $n=4$; Pergolide: $n=3$ ) and had never been treated with anticholinergic substances prior to the experiment.

\subsection{Procedure}

A double-blind, crossover method was used for both patients and normal controls, using acute administration of scopolamine and placebo. A fixed dose of $0.25 \mathrm{mg}$ s.c. of scopolamine was selected as an appropriate sub-clinical dose that does not produce central effects in normal controls $[6,13,50]$. Saline solution was used as placebo. The two substances were sequentially administered in a random order in two consecutive days. After complete description of the study to the subjects, written informed consent was obtained.

A Visual Analogue Scale (VAS) of subjective vigilance was administered immediately prior to and 30 minutes after the injection. In this scale, subjects had to evaluate their level of vigilance by making a dash with a pencil along a $100 \mathrm{~mm}$ line indicating 'drowsiness' at one end $(0 \mathrm{~mm})$ and 'good alertness' at the other $(100 \mathrm{~mm})$. The score was the distance in $\mathrm{mm}$ from the zero extremity to the dash. Variations of vigilance induced by scopolamine and by placebo were expressed by the ratio of the post-injection score over the pre-injection score (expressed in percentage).

Neuropsychological testing began just after the second VAS evaluation and included:

- The Grober \& Buschke Verbal Memory Task $[23,24]$, in which subjects were required to learn a list of 16 words each associated with a specific semantic category (e.g., animal, furniture,...). Subjects were first asked to make a free recall of these words. A cued recall was then carried out, where the examiner gave semantic categories corresponding to words that were not recalled. This procedure was repeated three successive times and followed, 20 minutes later, by a delayed recall that includes a free recall, a cued recall and a recognition task.

- A Word Repetition Priming Task, was administered as an evaluation of implicit memory. This test was not presented as a memory test to the subjects. Sixteen words were presented with the instruction of making an affective judgment on each of them. Five minutes later, subjects were asked to complete a list of 48 syllables with the first word that comes to mind. Sixteen of these syllables came from the list of words presented previously. After this implicit recall, subjects were first asked to explicitly recall the 16 words of the list, and then asked to recognize target words among distractors.

- The SelfOrdered Pointing Task (SOPT) [39], consists of a stack of sheets on which a set of $6,8,10$ or 12 abstract designs were displayed. For each stack of sheets, the abstract designs remained the 
sames but their relative position varied at random from sheet to sheet. The subject's task was to go through the stack, touching only one design on each sheet while taking care not to touch the same design twice. The subject himself initiated the program and determined the order of responding. Each set of designs $(6,8,10,12)$ were administered three consecutive times. The score was the total number of errors (defined as pointing a design that had already been pointed in a given set). The test is considered to be a good measure of resistance to proactive interference and of visual working-memory.

- The Shifting Reaction Time Task [7] was an adaptation of Posner's paradigm of covert orienting of attention [44]. In this choice reaction time task, subjects were asked to fix their gaze on a central point shown on a computer screen and instructed to press a response button as quickly as possible with the right or left index finger depending on the side of target presentation on screen. The target (a red square) appeared in a random sequence, in a $50 \%$ ratio to each side of the central fixation point. Prior to the target $(350 \mathrm{~ms})$, a cue was presented for $200 \mathrm{~ms}$ at the central fixation point. This succession of cue, target and response was considered as a trial, and an experimental session included 300 trials. For one third of the trials, the visual cue consisted of a cross $\left(1 \mathrm{~cm}^{2}\right)$ indicating that the target was equally likely to occur to the left or to the right. This was the neutral cue. On the other two thirds of trials, the cue consisted of an arrow $(0.5 \mathrm{~cm} \times 2 \mathrm{~cm})$ pointing to the left or to the right, indicating the most probable side to which the target would be delivered. Valid cues (80\% of the arrows) consisted of correct indications of target, while invalid cues $(20 \%$ of the arrows) were indications which incorrectly predicted the side of the target. Mean reaction times (RTs) were collected separately for the neutral, valid and invalid cue trials.

In PD, as in normal subjects, targets correctly cued (Valid) are usually detected faster than uncued (Neutral) or incorrectly cued (Invalid) targets. However, during invalid cue trials, PD patients, but not control subjects, make frequent errors (response to the cued side rather than to the stimulated side) [7]. In addition, RT of these errors during invalid cue trials are usually as short as during valid cue trials, suggesting that the mental operations associated to the shifting from the cued to the stimulated side do not occur. For these reasons, errors made during invalid cue trials were considered to be shifting errors.

\subsection{Statistical analysis}

The effects of scopolamine and placebo on the vigilance VAS, the Word Repetition Priming Task and the SOPT were compared between normal controls and parkinsonian patients using multiple analysis of variance (ANOVA) for repeated measures including one between-groups factor (Group) and one within-group factor (Treatment). Significant differences were further contrasted using Student $t$-tests with Bonferroni adjusted significance level for multiple comparisons. ANOVAs were performed separately for the Grober \& Buschke Verbal Memory Test and the Shifting RT Task including one between-groups factor (Group) and two within-group factors (Treatment $\times$ Trial $\left[1^{\text {st }}, 2^{\text {nd }}, 3^{\text {rd }}\right.$ recalls] for the Grober \& Buschke and Treatment $\times$ Cue condition [valid, neutral, invalid] for the Shifting RT Task). ANOVA for repeated measures were then carried out on the specific significant factors. Errors in the Shifting RT Task were computed separately using ANOVA for repeated measures that includes one between (Group) and one within (Treatment) group factors.

\section{Results}

In normal controls as in parkinsonian patients, subjective level of vigilance (VAS) did not differ between placebo and scopolamine treatment, since there were no group effect $[f(1,18)=0.02$, N.S.], no treatment effect $[f(1,18)=0.12$, N.S. $]$, and no group by treatment interaction $[f(1,18)=0.79$, N.S.]. Most subjects evaluated their level of vigilance similarly during pre- and post-injection periods. In normal controls, the mean post-injection score during placebo was of $92.8 \%( \pm 5.9)$ the pre-injection score, while during scopolamine it was of $90.1 \%( \pm 7.9)$. In parkinsonian patients, these scores were of $90.9 \%( \pm 6.7)$ during placebo and of $92.1 \%( \pm 8.4)$ during scopolamine.

Fig. 1 shows the learning performance of both groups in the Grober \& Buschke Verbal Memory Test. In the free recall, ANOVA reveals significant group effect $[f(1,18)=15.7, p<0.01]$, treatment effect $[f(1,18)=32.6, p<0.01]$, trial effect $[f(2,36)=$ $104.8, p<0.001]$ and a significant group by treatment by trial interaction $[f(2,36)=20.6, p<0.01]$. Therefore, further analyses were performed separately 
IMMEDIATE FREE RECALLS
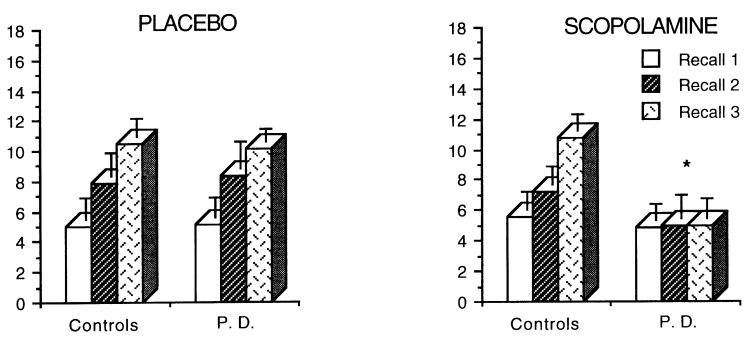

IMMEDIATE TOTAL RECALLS
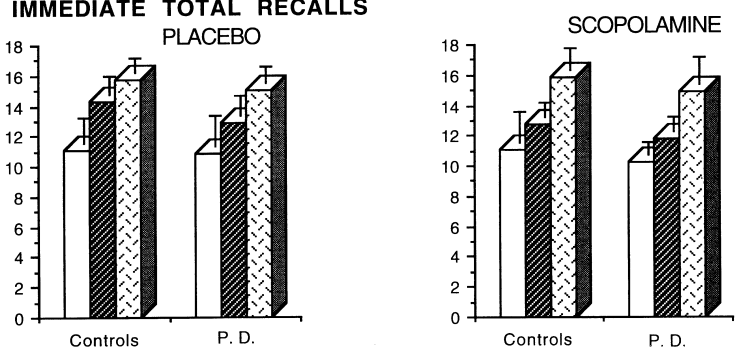

Fig. 1. Grober \& Buschke verbal memory test.

for placebo and scopolamine conditions. During placebo, free recall was comparable between the two groups (no group effect: $[f(1,18)=0.01$, N.S.], no group $\times$ trial interaction: $[f(2,36)=0.6$, N.S.]), with a progressive learning over the three successive trials (trial effect: $[f(2,36)=98.1, p<0.01]$ ). However, during scopolamine treatment, the performances differed between the two groups (group effect: $[f(1,18)=41.9, p<0.01]$, group $\times$ trial interaction: $[f(2,36)=46.3, p<0.01])$, in that controls still showed a progressive learning among trials (trial effect: $[f(2,18)=67.9, p<0.01])$ while parkinsonian patients no longer showed the learning effect $[f(2,18)=0.34$, N.S. $]$. In the total recall (summation of free and cued recalls), the two groups showed the same pattern of performance (see Fig. 1) with a significant trial effect $[f(2,36)=83.4, p<0.01]$, without group effect $[f(1,18)=0.47$, N.S. $]$ nor treatment effect $[f(1,18)=1.3$, N.S. $]$.

ANOVA performed on the delayed recall of the Grober \& Buschke Memory Test (Fig. 2) showed a group effect $[f(1,18)=72.4, p<0.001]$, a treatment effect $[f(1,18)=49.9, p<0.001]$ and a group by treatment interaction $[f(1,18)=81.7, p<0.001]$ during the free recall, but not during the total recall $([f(1,18)=1.05$, N.S. $],[f(1,18)=0.14$, N.S.], $[f(1,18)=0.98$, N.S.] respectively). During placebo, Student $t$-tests revealed that there were no difference between groups in both free recall $[t(18)=$ 1.1, N.S.] and total recall $[t(18)=0.06$, N.S.]. $\begin{array}{cll}\text { DELAYED RECALL } & \square \text { Free Recall } \\ \text { PLACEBO } & \mathbb{Q} \text { Total Recall } \\ \text { SCOPOLAMINE }\end{array}$
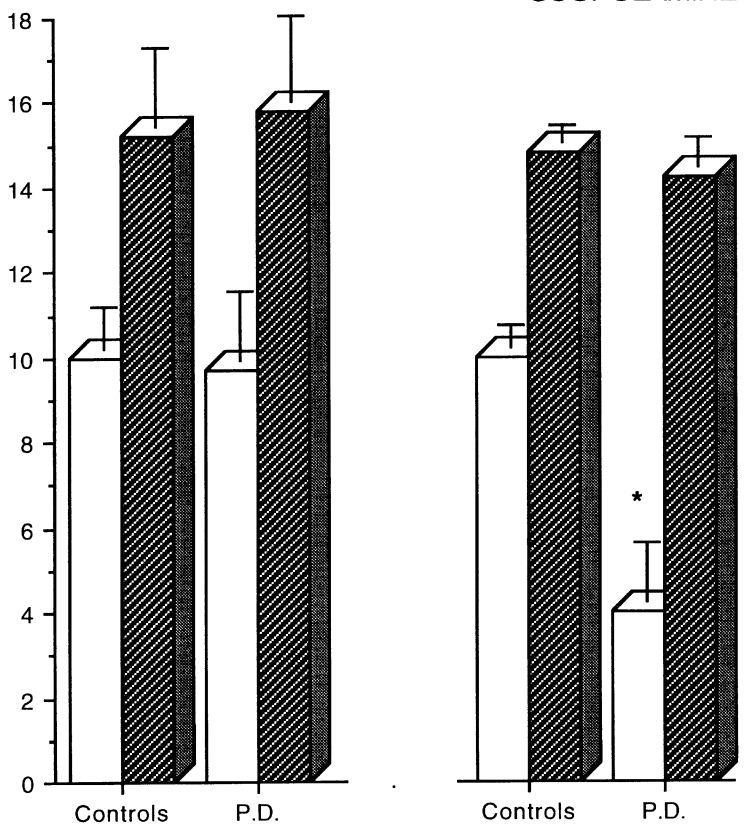

Fig. 2. Grober \& Buschke verbal memory test.

However, during scopolamine treatment, parkinsonian patients were lower than controls in free recall $[t(18)=11.3, p<0.001]$, while total recall still remain comparable between groups $[t(18)=1.01$, N.S.]. Finally, recognition was similar between the two groups both during placebo $[t(18)=0.6$, N.S.] and during scopolamine treatment $[t(18)=0.7$, N.S.], with all subjects except one recognising the 16 words.

In the Word-Repetition Priming Test (See Fig. 3) there were no group effect $[f(1,18)=0.8$, N.S.], no treatment effect $[f(1,18)=1.4$, N.S.], and no group by treatment interaction $[f(1,18)=1.0$, N.S.] during the Completion part. The same has been observed in the Recognition part of this test (group: $[f(1,18)=0.5$, N.S. $]$, treatment: $[f(1,18)=1.8$, N.S. $]$, group $\times$ treatment: $[f(1,18)=2.6$, N.S. $])$. However, in the Free Recall, there were a group effect $[f(1,18)=14.7, p<0.001]$, a treatment effect $[f(1,18)=12.8, p<0.001]$ and a group by treatment interaction $[f(1,18)=20.0, p<0.001]$. Contrasts with Student $t$-tests indicate that these effects were attributable to similar performances between groups during placebo $[t(18)=1.8$, N.S.], and different performances during scopolamine treatment $[t(18)=12.7$, $p<0.001]$. In the SOPT (See Fig. 4), group by treatment interaction was also observed $[f(1,18)=6.4$, 


\section{COMPLETION}

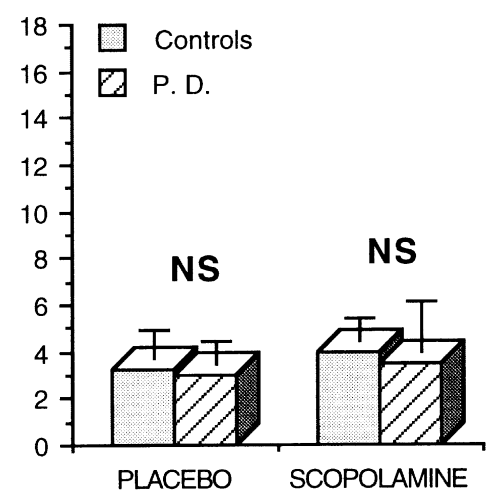

\section{FREE RECALL}
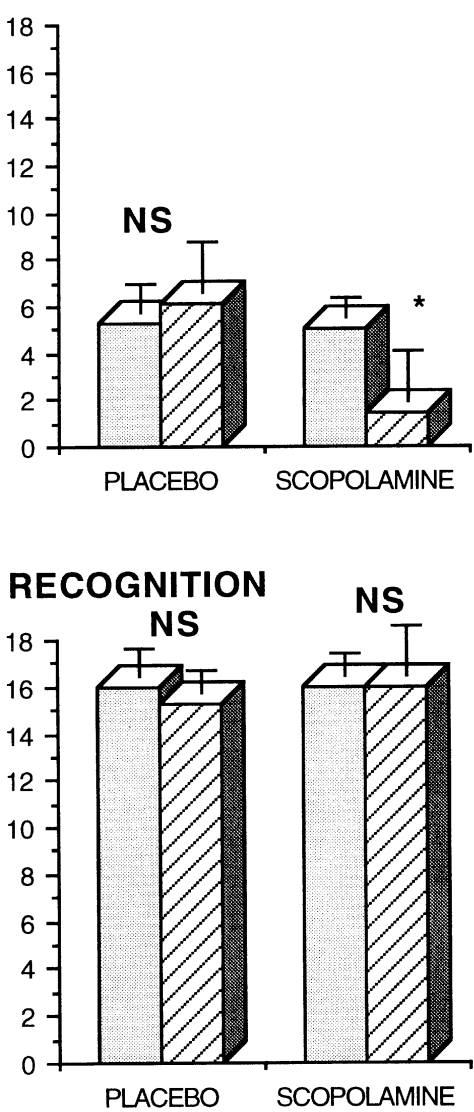

Fig. 3. Word repetition priming task.

$p<0.05]$, with a number of interference errors that did not differ between groups during placebo $[t(18)=0.9$, N.S.], but did differ during scopolamine treatment $[t(18)=9.2, p<0.05]$.

In the Shifting Reaction Time task (Fig. 5), ANOVA on RT values (excluding RT during errors) revealed a

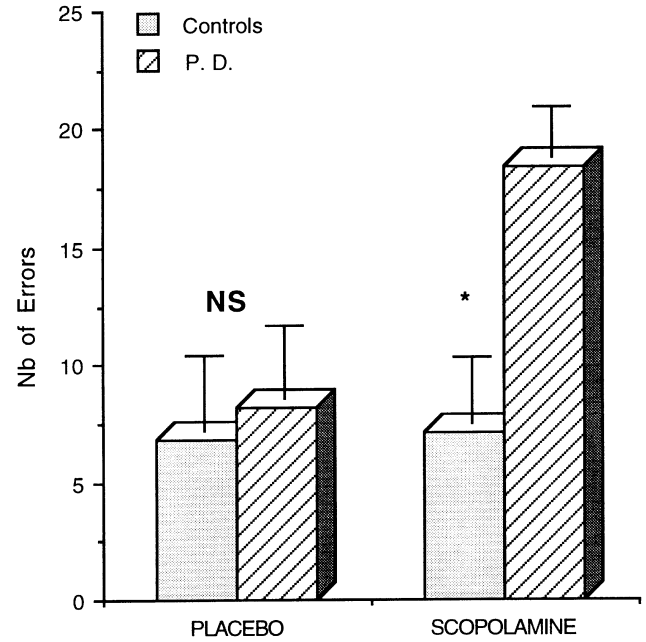

Fig. 4. Self ordered pointing task.
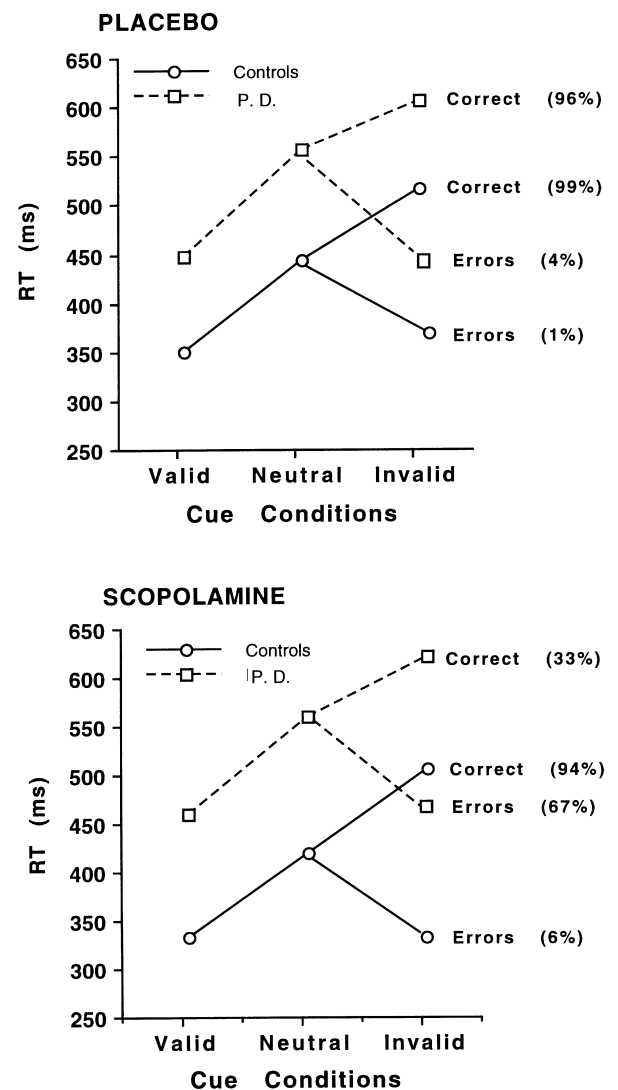

Fig. 5. Shifting reaction time task.

significant group effect $[f(1,18)=10.1, p<0.01]$ and a significant cue effect $[f(2,36)=11.8, p<$ $0.01]$, but no treatment effect $[f(1,18)=2.7$, N.S. $]$ and no interactions $($ group $\times$ cue: $[f(2,36)=1.8$, 
N.S.], treatment $\times$ cue $[f(2,36)=1.0$, N.S.], group $\times$ treatment $\times$ cue $[f(2,36)=0.4$, N.S.]). This suggests that the performance was not affected by treatment and that both groups had the same RT profile during valid, neutral and invalid cue trials, even though parkinsonian patients were significantly slower than normal controls (mean differences of $110 \mathrm{~ms}$ ) in each condition (See Fig. 5). ANOVA performed specifically on the number of shifting errors revealed a significant group effect $[f(1,18)=5.2, p<0.05]$, a significant treatment effect $[f(1,18)=8.0, p<0.05]$ and a significant group by treatment interaction $[f(1,18)=12.3$, $p<0.01]$. Errors made during placebo appear greater in parkinsonians $(4 \%)$ than in controls $(1 \%)$, but this slight non significant tendency $[t(18)=2.1$, N.S.] was artificially created by a patient who makes multiple impulsive errors during invalid as well as during neutral cue trials. However, during the scopolamine treatment, the number of shifting errors in parkinsonian patients was significantly greater $(67 \%)$ than in normal controls $(6 \%)[t(18)=11.2, p<0.01]$.

\section{Discussion}

As previously suspected $[6,13]$, this experiment showed that the administration of scopolamine at a subclinical dose may induce a transient SCFS in parkinsonian patients. The nature of the deficits in the Grober \& Buschke and Words Repetition Priming memory tasks suggests that there was no genuine amnesia. In PD, but not in controls, free recall was affected by scopolamine. However, when semantic cues were given to parkinsonian patients, their performances improved and the total recall of words became similar to the one of the controls. Recognition and completion indicate preserved capacities of retention. In other words, there was no loss of information during scopolamine treatment, but rather a recall difficulty similar to the one observed in SCFS [42, 43], and attributable to deficiencies in recollection strategies. Other results of the present study consistent with a transient SCFS during scopolamine treatment, were the number of errors in both the SOPT and the Shifting RT Task, that suggests an excessive sensitivity to interference and a shifting deficit, respectively.

The simultaneous induction of shifting deficits, proactive interference and characteristic deficiencies in strategies of recollection - three cardinal features of SCFS - may indicate a direct action of anticholinergics on the central physiological and cognitive sub- stratum of the syndrome. From a physiological point of view, such an effect obtained with a subclinical dose of scopolamine suggests a cancelling action of the drug on cholinergic compensatory mechanisms $[1,4]$ that seems to take place in PD. In other words, compensatory mechanisms of cholinergic depletion in PD may no longer be robust enough to withstand agents aimed at blocking muscarinic receptor sites, even at a subclinical dose.

From a cognitive point of view, our results also suggest an action on the basic processes underlying SCFS. The Supervisory Attentional System (SAS) - a conceptual framework taken from cognitive psychology $[38,48]$ - has been proposed as a possible basic process of which a malfunctioning might induce SCFS in PD $[8,9,12]$. SAS is viewed as a limited capacity attentional system responsible for controlling nonroutine mental activity, and therefore called upon only under certain situations. For instance, recognition is a passive memory situation in which SAS does not intervene to mentally scan memories, establish a strategic search, inhibit interferences and select the appropriate mnestic material, while such mental works are necessary during free recall and thus require the SAS.

A general SAS impairment such as the one suspected in PD may result from both a depletion of attentional resources, and a difficulty in strategic allocation of these attentional resources over the multiple cognitive components implicated in information processing $[8,9]$. However, the problem with the concept of 'attentional resources' remains its quantification. Allocation of resources is usually assessed by performances in tasks requiring the processing of concurrent information $[9,12,34]$. However, the quantity of attentional resource always remains difficult to estimate. Since the concept refers to energy that may activate different cognitive processes, one may suggest that the level of arousal could be an indirect quantitative estimation of resources. It is therefore possible that the scopolamine induced SCFS in PD is mainly an arousal-dampening effect, given that this substances can facilitate sedation. This would be in accordance with other studies suggesting that parkinsonian patients have difficulties with tasks requiring alertness and effort [56]. This would also follow the idea that anticholinergics deplete the cognitive processing resources, such as the amount of mental effort which can be applied to neuropsychological tasks [57]. Since executive tasks are usually more effortful than the others, they would most suffer from a reduction in mental resources. 
In this respect, the present study has shown that subjective vigilance did not differ between placebo and scopolamine treatment both in normal controls and in parkinsonian patients. Moreover, RT values in the Shifting RT task were not different between placebo and scopolamine treatment, suggesting no variation of arousal. Therefore, if attentional resources are a matter for the level of arousal, it is unlikely that scopolamine induced SCFS in PD result from a reduction of attentional resources. Consequently, deficient allocation of attentional resources could potentially be the basic cognitive cholinergic-dependent mechanism underlying SCFS in PD.

\section{Acknowledgements}

The authors which to thank Drs Michèle Morin and Hélène Pereault for their medical assistance during the experiment. This work was supported by the 'Fonds de la Recherche en Santé du Québec'.

\section{References}

[1] Y. Agid, F. Javoy-Agid and M. Ruberg, Biochemistry of neurotransmitters in Parkinson's Disease, in: Movement disorders II, C.D. Marsden and S. Fahn, eds, Butterworths \& Co. Publishers, London, 1987, pp. 166-230.

[2] Y. Agid, M. Ruberg, F. Javoy-Agid, E. Hirsch, R. RaismanVozari, S. Vyas, B. Faucheux, P. Michel, A. Kastner, V. Blanchard, P. Damier, J. Villares and P. Zhang, Are dopaminergic neurons selectively vulnerable to Parkinson's disease?, in: Advances in Neurology, Vol. 60, H. Narabayashi, T. Nagatsu, N. Yanagisawa and Y. Misuno, eds, Raven Press, NewYork, 1993, pp. 148-164

[3] G.E. Alexander, M.R. DeLong and P.L. Strick, Parallel organization of functionally segregated circuits linking basal ganglia and cortex, Annual Review of Neuroscience 9 (1986), 357-381.

[4] M. Asahina, H. Shinotoh, K. Hirayama, K. Suhara, F. Shishido, O. Inoue and Y. Tateno, Hypersensitivity of cortical muscarinic receptors in Parkinson's disease demonstrated by PET, Acta Neurologica Scandinavica 91 (1995), 437-443.

[5] J. Beardsley and F. Puletti, Personality (MMPI) and cognitive (WAIS) changes after levodopa treatment, Archives of Neurology 25 (1971), 145-150.

[6] M.A. Bédard, H. Masson, H. Perreault and S. Gaudreau, Specific memory deficits related to lesions of the cholinergic systems in Parkinson's disease, in: Dementia in Parkinson's disease, A.D. Korczyn, ed., Monduzzi Editore, Bologna, Italy, 1994, pp. 213-216

[7] M.A. Bédard, F. El Massioui, C. Malapani, B. Dubois, B Pillon, B. Renault and Y. Agid, Attentional deficits in Parkinson's disease: Partial reversibility with Naphtoxazine (SDZ NVI-085), a selective noradrenergic $\alpha_{1}$ agonist, Clinical Neuropharmacology 21 (1998), 108-117.
[8] R.G. Brown and C.D. Marsden, Internal versus external cues and the control of attention in Parkinson's disease, Brain 111 (1988), 323-345.

[9] R.G. Brown and C.D. Marsden, Dual task performance and processing resources in normal subjects and patients with Parkinson's disease, Brain 114 (1991), 215-231.

[10] J.A. Cooper, H.J. Sagar, S.M. Doherty, N. Jordan, P. Tidswell and E.V. Sullivan, Different effects of dopaminergic and anticholinergic therapies on cognitive and motor function in Parkinson's disease, Brain 115 (1992), 1701-1725.

[11] J.L. Cummings, Subcortical Dementia, Oxford University Press, New-York, 1990.

[12] J.C. Dalrymple-Alford, A.S. Kalders, R.D. Jones and R.W. Watson, A central executive deficit in patients with Parkinson's disease, Journal of Neurology, Neurosurgery and Psychiatry 57 (1994), 360-367.

[13] B. Dubois, F. Danzé, B. Pillon, G. Cusimano, F. Lhermitte and Y. Agid, Cholinergic-dependent cognitive deficits in Parkinson's disease, Annals of Neurology 22 (1987), 26-30.

[14] B. Dubois, B. Pillon, F. Lhermitte and Y. Agid, Cholinergic deficiency and frontal dysfunction in Parkinson's disease, Annals of Neurology 28 (1990), 117-121.

[15] B. Dubois and B. Pillon, Biochemical correlates of cognitive changes and dementia in Parkinson's disease, in: Parkinson's disease: neurobehavioral aspects, S.J. Huber and J.L. Cumming, eds, Oxford University Press, Oxford, 1992, pp. 178198.

[16] N. Duchesne, M.A. Bédard, J.P. Soucy and H. Masson, Relationship between the deficit in sharing attention and the degree of striatal dopaminergic denervation in Parkinson's disease: A SPECT study using IODINE 123-B-CIT, Neurology 50 (1998), A44.

[17] C. Duyckaerts, P. Gaspas, C. Costa, A.M. Bonnet and J.J. Hauw, Dementia in Parkinson's disease-Morphometric data, in: Advances in Neurology, Vol. 60, H. Narabayashi, T. Nagatsu, N. Yanagisawa and Y. Misuno, eds, Raven Press, NewYork, 1993, pp. 447-455.

[18] M.F. Folstein, S.E. Folstein and P.R. McHugh, Mini-mental state: a practical method for grading the cognitive state of patients for the clinician, Journal of Psychiatric Research 12 (1975), 189-198.

[19] R.B. Godwin-Austen, E.B. Tomlinson, C.C. Frears and H.W.L. Kok, Effects of L-Dopa in Parkinson's disease, Lancet ii (1969), 165-168.

[20] P.S. Goldman-Rakic, Circuitry of the primate prefrontal cortex and the regulation of behavior by representational memory, in: Handbook of physiology: The nervous system, higher functions of the brain, Section 1, Vol. V (part 1), F. Plum, ed., American Physiology Society, Bethesda, 1987, pp. 373-417.

[21] A.M. Gotham, R.G. Brown and C.D. Marsden, 'Frontal' cognitive function in patients with Parkinson's disease 'on' and 'off' levodopa, Brain 111 (1988), 299-321.

[22] E. Grober and H. Buschke, Genuine memory deficits in dementia, Developmental Neuropsychology 3 (1987), 13-36.

[23] E. Grober, H. Buschke, M.D. Crystal, M.A. Bang and R. Dresner, Screening for dementia by memory testing, Neurology 38 (1988), 900-903.

[24] E. Grober, R.M. Leipzig, R.B. Lipton, W. Wisniewski, M. Schroeder, P. Davies, W. Ritier and H. Buschke, Does scopolamine directly impair memory?, Journal of Cognitive Neurosciences 1 (1989), 329-335.

[25] E. Hirsch, A.M. Graybiel, C. Duyckaerts and F. Javoy-Agid, Neuronal loss in the pedunculopontine tegmental nucleus in Parkinson's disease and in Progressive Supranuclear Palsy, 
Proceedings of the National Academy of Sciences 84 (1987), 5976-5980.

[26] M.M. Hoehn and M.D. Yahr, Parkinsonism: onset, progression and mortality, Neurology 17 (1967), 427-442.

[27] O. Hornykiewicz, Parkinson's disease and the adaptive capacity of the nigrostriatal dopamine system: possible neurochemical mechanisms, in: Advances in Neurology, Vol. 60, H. Narabayashi, T. Nagatsu, N. Yanagisawa and Y. Misuno, eds, Raven Press, New-York, 1993, pp. 140-147.

[28] S.J. Huber, H.G. Shulman, G.W. Paulson and E.C. Shuttleworth, Fluctuations in plasma dopamine level impair memory in Parkinson's disease, Neurology 37 (1989), 1371-1375.

[29] F. Javoy-Agid and Y. Agid, Is the mesocortical dopaminergic system involved in Parkinson's disease?, Neurology 30 (1980), 1326-1330.

[30] K. Jellinger, The pedunculopontine nucleus in Parkinson's disease, progressive supranuclear palsy and Alzheimer's disease, Journal of Neurology, Neurosurgery and Psychiatry $\mathbf{5 1}$ (1988), 540-543.

[31] K.W. Lange, T.W. Robbins, C.D. Marsden, M. James, A.M Owens and G.M. Paul, L-dopa withdrawal in Parkinson's disease selectively impairs cognitive performance in tests sensitive to frontal lobe dysfunction, Psychopharmacology Berlin 107(2-3) (1992), 394-404

[32] M. Lezak, Neuropsychological assessement, 3rd ed, Oxford, New-York, 1995

[33] A.W. Loranger, H. Goodell, J.E. Le and F. McDowell, Levodopa treatment of Parkinson's syndrome improve intellectual functionning, Archives of General Psychiatry 26 (1972), $163-168$.

[34] C. Malapani, B. Pillon, B. Dubois and Y. Agid, Impaired simultaneous cognitive task performance in Parkinson's disease: A dopamine-related function, Neurology 44 (1994), 319-326.

[35] C. Malapani, B. Rakatin, R. Levy, W.H. Meck, B. Deweer, B. Dubois and J. Gibbon, Coupled temporal memories in Parkinson's disease: A dopamine-related dysfunction, Journal of Cognitive Neuroscience 10 (1998), 316-331.

[36] S. Mattis, Mental status examination for organic mental syndrome in the elderly patient, in: Geriatric Psychiatry, L. Bellak and T.B. Karasu, eds, Grune \& Stratton, New York, 1976 , pp. 77-121.

[37] E. Mohr, G. Fabbrini, J. Williams, J. Schlegel, C. Cox, P. Fedio and T.N. Chase, Dopamine and memory function in Parkinson's disease, Movement Disorders 4(2) (1989), 113 120.

[38] D.A. Norman and T. Shallice, Attention to action: willed and automatic control of behaviour, University of California CHIP Report 99, 1980.

[39] M. Petrides and B. Milner, Deficits on subject - ordered tasks after frontal and temporal lobe lesions in man, Neuropsychologia 20 (1982), 249-262.

[40] B. Pillon, B. Dubois, A.M. Bonnet, M. Esteguy, J. Guimaraes, J.M. Vigouret, F. Lhermitte and Y. Agid, Cognitive 'slowing' in Parkinson's disease fails to respond to levodopa treatment: the 'Fifteen objects test', Neurology 39 (1989a), 762-768.

[41] B. Pillon, B. Dubois, G. Cusimano, A. Bonnet, F. Lhermitte and Y. Agid, Does cognitive impairment in Parkinson's disease result from non-dopaminergic lesions?, Journal of Neurology, Neurosurgery, and Psychiatry 52 (1989b), 201-206.

[42] B. Pillon, B. Deweer, Y. Agid and B. Dubois, Explicit mem- ory in Alzheimer's, Huntington's and Parkinson's diseases, Archives of Neurology 50 (1993), 374-379.

[43] B. Pillon, N. Gouider-Khouja, B. Deweer, M. Vidailhet, C. Malapani, B. Dubois and Y. Agid, Neuropsychological pattern of striatonigral degeneration: comparison with Parkinson's disease and progressive supranuclear palsy, Journal of Neurology, Neurosurgery, and Psychiatry 58 (1995), 174179.

[44] M.I. Posner, Orienting of attention, Quaterly Journal of Experimental, Psychology 32 (1980), 3-25.

[45] J.M. Rabey, M.Y. Neufeld, T.A. Treves, P. Sifris and A.D. Korczyn, Cognitive effects of scopolamine in dementia, Journal of Neural Transmission: General section 103 (1996), 873-881.

[46] M. Ruberg, F. Rieger, A. Villageois, A.M. Bonnet and Y. Agid, Acetylcholinesterase and butylcholinesterase in frontal cortex and cerebrospinal fluid of demented and non-demented patients with Parkinson's disease, Brain Research 362 (1986), 83-91.

[47] J.S. Schneider and C.J. Kovelowski, Chronic exposure to low doses of MPTP. I. Cognitive deficits in motor asymptomatic monkeys, Brain Research 519 (1990), 122-128.

[48] T. Shallice, Specific impairments of planning, Philosophical transactions of the Royal Society of London B 298 (1982), 199-209.

[49] K.M. Shaw, A.J. Lees and G.M. Stern, The impact of treatment with levodopa on Parkinson's disease, Quaterly Journal of Medicine 49 (1980), 283-293.

[50] T. Sunderland, P.N. Tariot, R.M. Cohen, H. Weingartner, E.A. Mueller and D.L. Murphy, Anticholinergic sensitivity in patients with dementia of the Alzheimer type and agematched controls, Archives of General Psychiatry 44 (1987), 418-426.

[51] A.E. Taylor, J.A. Saint-Cyr and A.E. Lang, Frontal lobe dysfunction in Parkinson's disease, Brain 109 (1986), 845-883.

[52] A.E. Taylor, J.A. Saint-Cyr and A.E. Lang, Parkinson's disease: cognitive changes in relation to treatment response, Brain 110 (1987), 35-51.

[53] A.E. Taylor and J.A. Saint-Cyr, The neuropsychology of Parkinson's disease, Brain and Cognition 28 (1995), 281296.

[54] K.P.M. Van Spaendonck, H.J.C. Berger, M.W.I. Horstink, E.L. Buytenhuijs and A.R. Cools, Impaired cognitive shifting in Parkinsonian patients on anticholinergic therapy, Neuropsychologia 31 (1993), 407-411.

[55] B.H. Wainer and M.M. Mesulam, Ascending cholinergic pathways in the rat brain, in: Brain Cholinergic Systems, M. Steriade and D. Biesold, eds, Oxford University Press, Oxford, 1990, pp. 65-199.

[56] H. Weingartner, S. Burns, R. Diebel and P.A. LeWitt, Cognitive impairments in Parkinson's disease: distinguishing between effort-demanding and automatic cognitive processes, Psychiatry Research 11 (1984), 223-235.

[57] K.A. Wesnes and P.M. Simpson, Can scopolamine produce a model of the memory deficits seen in aging and dementia?, in: Practical aspects of memory: current research and issues, M.M. Gruneberg, P.E. Morris and R.N. Sykes, eds, John Wiley, New York, 1988.

[58] R.M. Zweig, W.R. Jankel, J.C. Hedreen, R. Mayeux and D.L. Price, The pedunculopontine nucleus in Parkinson's disease, Annals of Neurology 26 (1989), 41-46. 


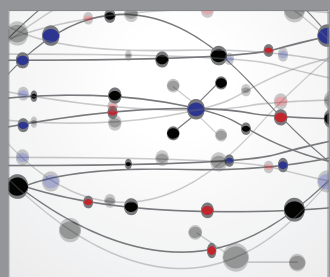

The Scientific World Journal
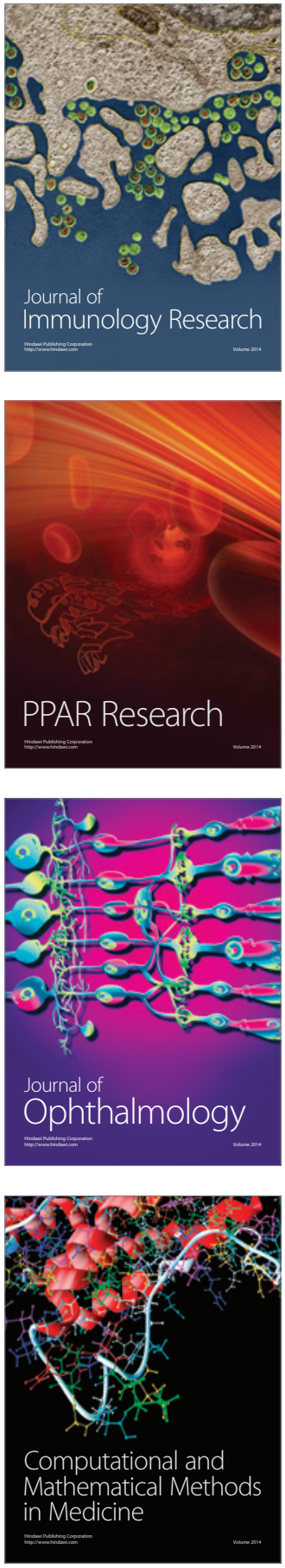



Gastroenterology

Research and Practice
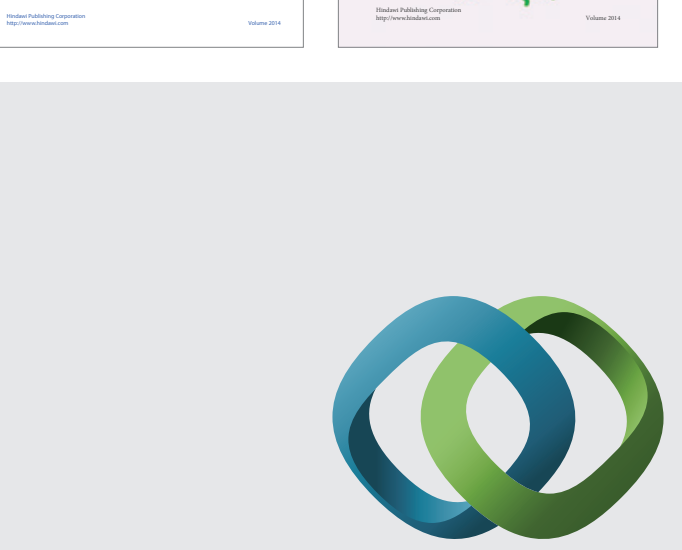

\section{Hindawi}

Submit your manuscripts at

http://www.hindawi.com
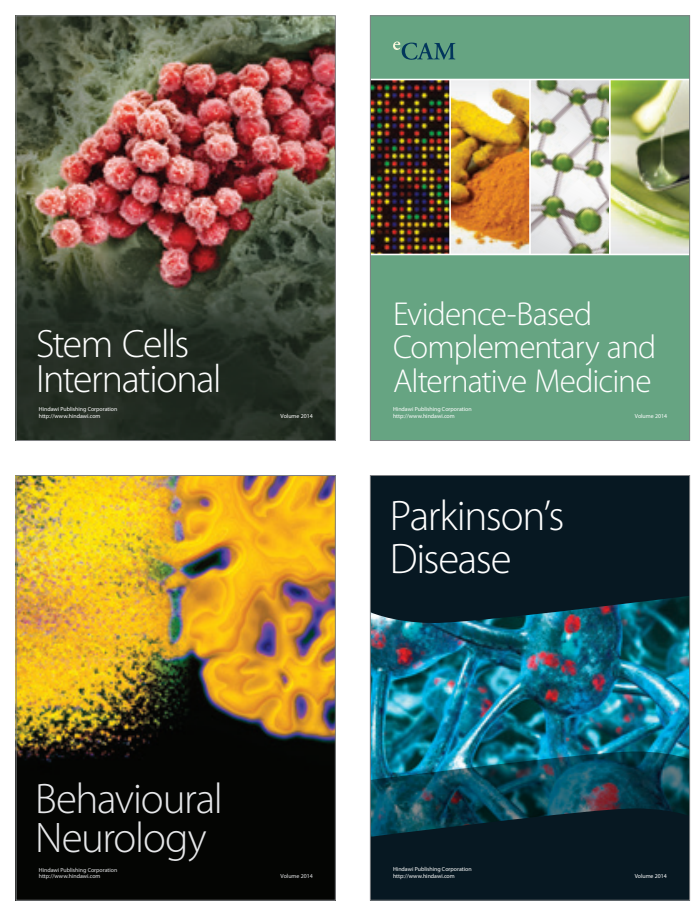

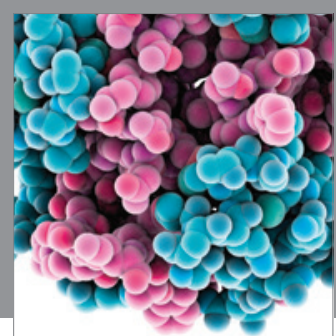

Journal of
Diabetes Research



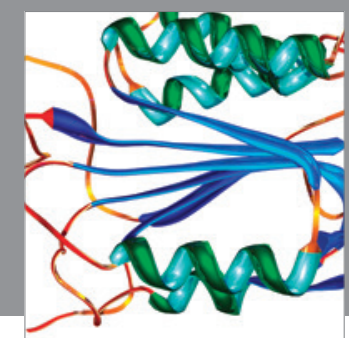

Disease Markers
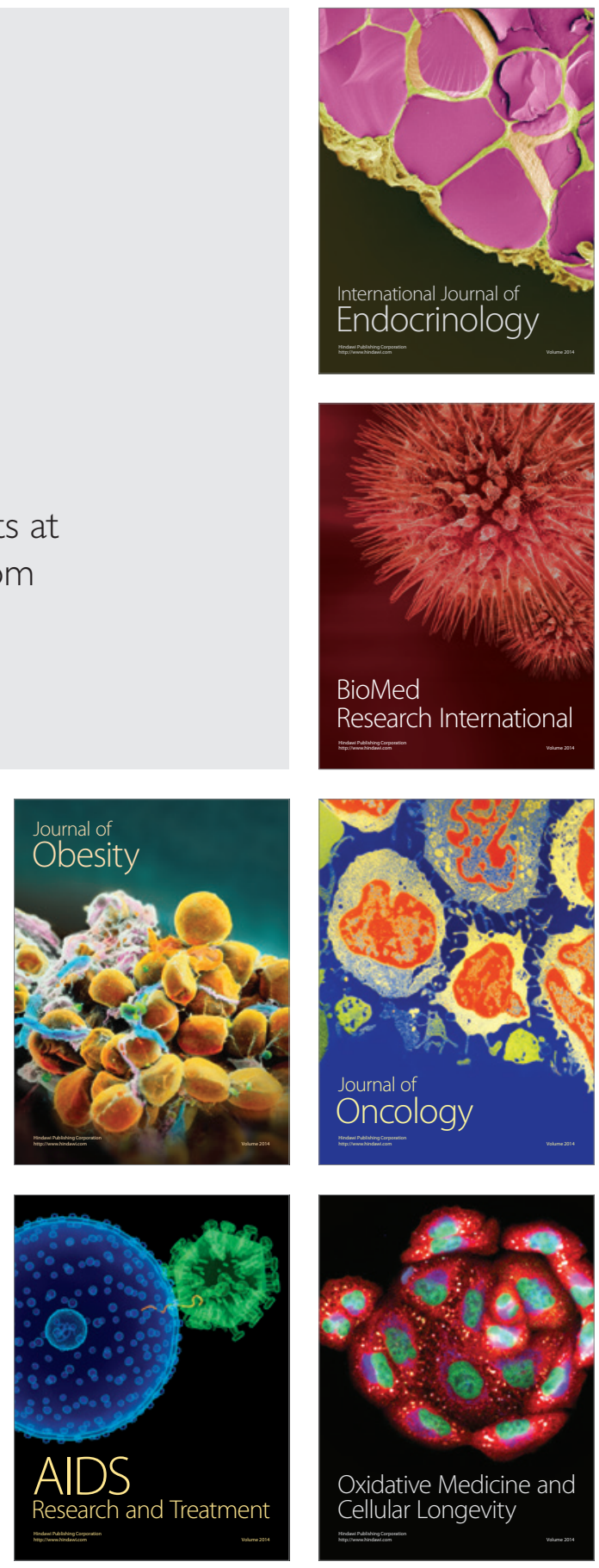